\title{
PHYSICAL SIMULATION OF THE INTEROSSEOUS LIGAMENTS DURING FOREARM ROTATION
}

\author{
Fabien Péan ${ }^{1 *}$, Fabio Carrillo ${ }^{2}$, Philipp Fürnstahl ${ }^{2}$, Orcun Goksel ${ }^{1}$ \\ 1,* Computer-assisted Applications in Medicine (CAiM), ETH Zurich, Switzerland, \\ fabien.pean@vision.ee.ethz.ch \\ ${ }^{2}$ Computer Assisted Research and Development Group, Balgrist University Hospital, \\ University of Zurich, Zurich, Switzerland
}

\section{INTRODUCTION}

The Interosseous Membrane (IOM) is a fibrous ligament bundle connecting the ulna and the radius. It is well known that the IOM allows transferring partial load from the radius to the ulna (Pfaeffle 2005). It also influences the kinematics of the radioulnar joint (Yasutomi 2002, Tarr 1984).

Three-Dimensional (3D) computer-assisted methods for preoperative planning of osteotomy have been applied successfully on forearm pathologies (Fürnstahl 2010, Murase 2008, Vlachopoulos 2015). However, to the best of our knowledge, models and studies of the influence of the IOM during pro-supination are limited to a kinematics analysis of the system, either with actual bone geometry (Fürnstahl 2009) or without it (Kasten 2002). In this work, we present a physical simulation of the forearm pro-supination involving the IOM biomechanical properties, for providing insights on the influence of the IOM on the radioulnar motion. We demonstrate a preliminary validation using a sample simulation performed on a healthy forearm by comparing its outcome with literature, and analyze the kinetic data that our method allows.

\section{MATERIALS AND METHODS}



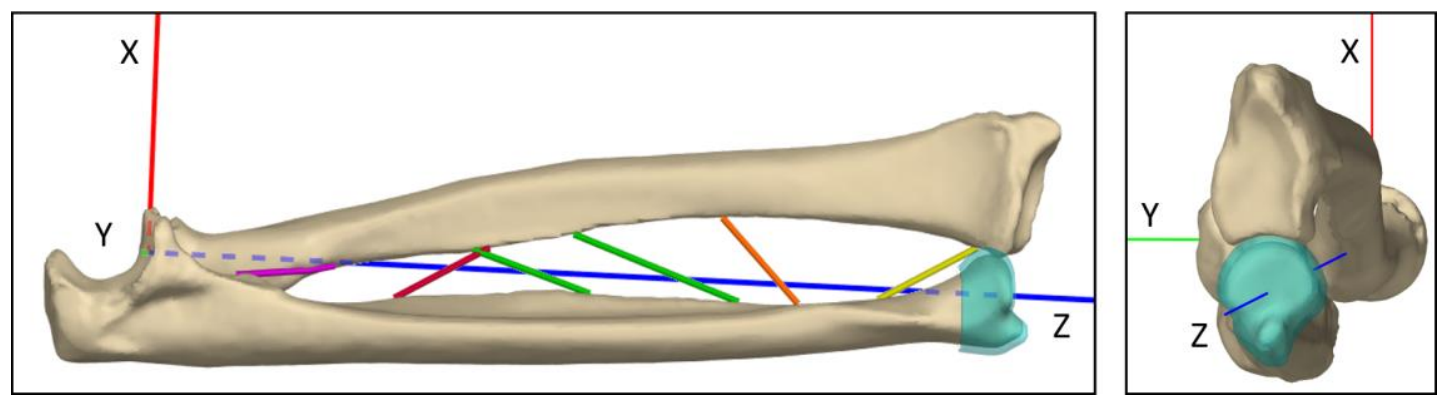

Figure 1: To the left, the palmar view of the ulna (bottom) and radius (top) connected by 6 ligament parts, from left to right: POB, DOAC, proximal CB, distal CB, distal AB, DOB. To the right, the distal radius and ulna in neutral position. The turquoise element around the ulna head emulates the cartilage. $Z$ is axis of rotation, $X$ the direction defined by the radius styloid and the ulna fovea projected onto the plane normal to $\mathrm{Z}$.

\section{Data Acquisition and Annotation}

Computed tomography (CT) scans of a cadaver specimen of a healthy left forearm were acquired with a Philips Brilliance 64 CT device with a slice thickness of $1 \mathrm{~mm}$. Radius and ulna were segmented in the image volume using the global thresholding and region growing functions in MITK (DKFZ, Heidelberg, Germany).

In this work, we modelled 5 ligaments in the IOM: the Distal Oblique Band (DOB), the Central Band (CB), the Accessory Band (AB), the Dorsal Oblique Accessory Cord (DOAC), the Proximal Oblique Cord (POC). Bone entry locations of these ligaments, as defined by (Noda 2009), were annotated on the segmented forearm surfaces. The axis of rotation was defined by the line going through the center of the proximal radial head and the distal ulna fovea.

An artificial cartilage model was generated since cartilage is not visible in CT. It can be assumed that, in the presence of cartilage, radius and ulna are in contact in the distal radioulnar joint. Thus, to simulate the cartilage, we defined a contact surface around the ulnar head by expanding it by a margin along its surface normals to bring it in contact with the radius. This allows the radius to rotate around the ulna assuming a fixed cartilage thickness.

\section{Simulation}

The simulation is performed in a biomechanical simulation toolkit Artisynth (Lloyd 2012). The motion from full pronation to full supination were simulated by rotating the radius from -110 to 110 degrees w.r.t. the neutral position around the annotated axis of rotation. In order to avoid over-constraining the kinematics of the radius, the movement is prescribed on a separate frame elastically coupled to the radius 
coordinate frame, which is located at the radius center of rotation. This achieves enforcing the rotation as a desired target, rather than a rigid constraint.

The proximal radiohumeral joint is defined as a ball-socket joint with compliant constraints. Distal radioulnar contact at the cartilage is modeled by stiff elastic collision constraints. A full backward Euler time integrator is employed to ensure stability.

A common ligament model is chosen, with no force when relaxed (below a set rest length) and a linear spring behaviour when engaged. We use the ligament stiffness from (Pfaeffle 1996) as $130 \mathrm{~N} / \mathrm{mm}$ for the Central Band. Since other ligament values are scarce in the literature, we approximate them by scaling the stiffness from CB to the ratio of their cross-sections using anatomical data reported in (Noda 2009). Thus, giving the following stiffnesses: $66 \mathrm{~N} / \mathrm{mm}$ for DOB, $41 \mathrm{~N} / \mathrm{mm}$ for POB, $29 \mathrm{~N} / \mathrm{mm}$ for DOAC. AB cross-section is absent from that study, although reported as thin; so it is set to the same value as the DOAC. Viscoelasticity does not apply since high-speed movement is not considered. Rest lengths of the ligaments have been rarely reported in the literature. Nevertheless, the IOM is known to be stiff, hence the rest lengths can be assumed to be the maximum length found in the kinematic analysis of the joint over the standard range of motion, which we used to set the rest lengths. We modeled collisions of the ligaments with the bones to enable the wrapping around the bones.

\section{RESULTS}

In Figure 2, the simulations were run repeatedly while incrementally activating some features. For comparison, the results reported in (Moritomo 2009) are superimposed on our figures with error bars representing the trend obtained from imaging observations of 9 healthy forearms. The results have been normalized with respect to the length at the neutral position.

Note that the trends are in agreement between our results and observations of (Moritomo 2009). The DOAC is the only ligament that does not follow strictly the (statistically significant) trend. A sensitivity analysis on the DOAC origin and insertion did not bring the trend any closer. One explanation is that the cadaver did not have the DOAC (present, e.g., only in 52\% of sample in (Noda 2009)), the presence of which was not recorded in this retrospective study. Moreover, anatomical differences can explain the dissimilarity since our specimen comes from a different ethnic group. 


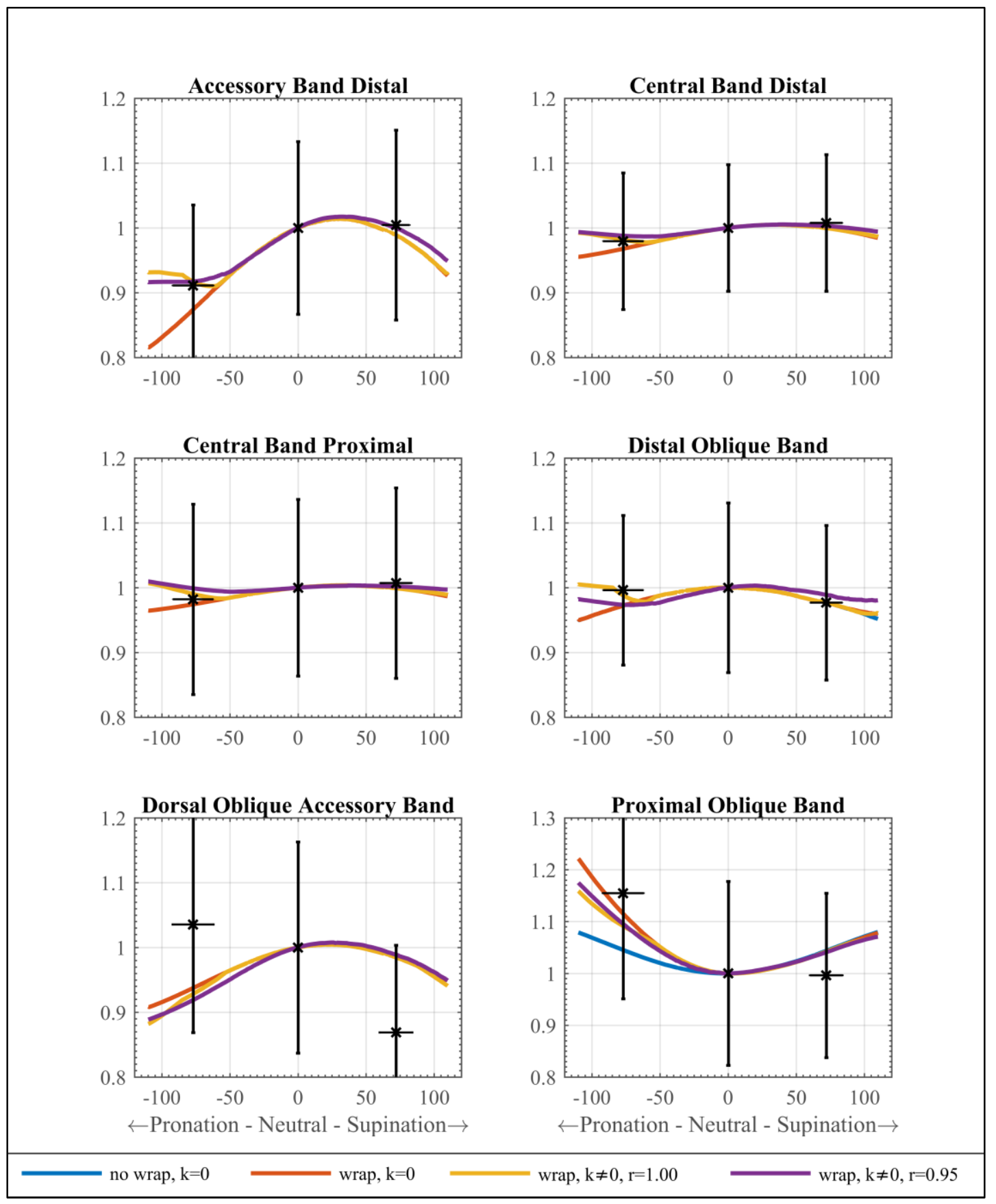

Figure 2: The normalized length of ligaments with respect to neutral length is shown over pro-supination for multiple incremental features activated: in blue, no ligament-bone collision (no wrap) and no stiffness in ligaments $(k=0)$; in red, ligament-bone collisions enabled (wrap); in yellow, ligament response enabled $(k \neq 0)$ with rest lengths set to the maximum length defined above $(r=1.00)$; in purple, rest lengths set at $95 \%$ of the maximum length to ensure pretensed ligaments ( $r=0.95$ ). Results from (Moritomo 2009) shown in black. 
As expected, the Figure 3 shows the ligament wrapping mainly affecting the POB response during pronation. If we plot the wrapped simulation instances as below, we note that DOB also wraps around the ulna in late supination.
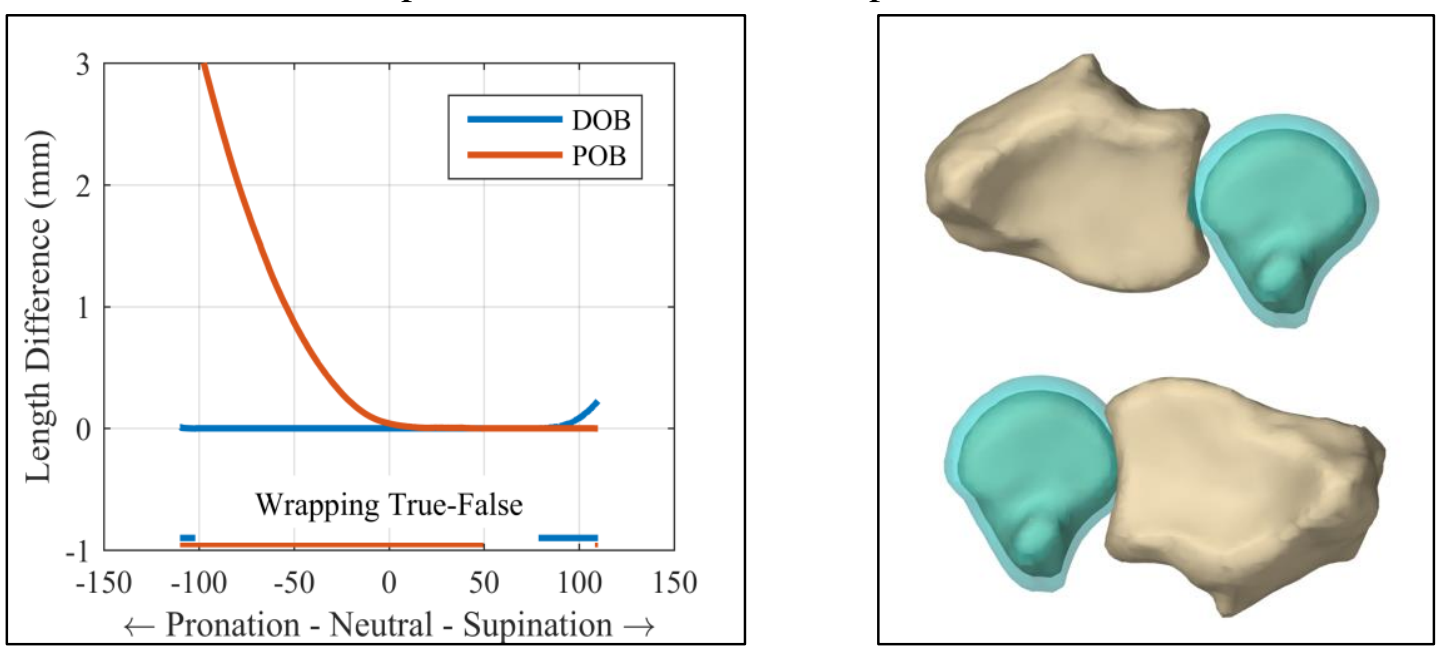

Figure 3: On the left, occurrence and effect of wrapping without accounting for ligament physical properties. On the right, pronation at 90 degree (top) and supination at 90 degree (bottom).

There is no clear difference between using no ligament model and with ligament forcemodel with rest length equal to neutral length. This is because, during the frame rotation, the ligaments lengths never go above the rest length over entire prosupination, thus not constraining the radius additionally. Nevertheless, when the rest length of the ligaments is set as $95 \%$ of their maximal length, the difference is noticeable, particularly for the ones on the distal part of the radius, the DOB and the $\mathrm{ABD}$, where the effect of additional ligament length constraints aggravates the most given the fixed humeral end.

The study of the landmarks located on the distal radius can indicate the influence of the IOM on the positions of the radius over time (see Figure 4). Changing the rest lengths completely modifies the evolution of the position of the radius over time.
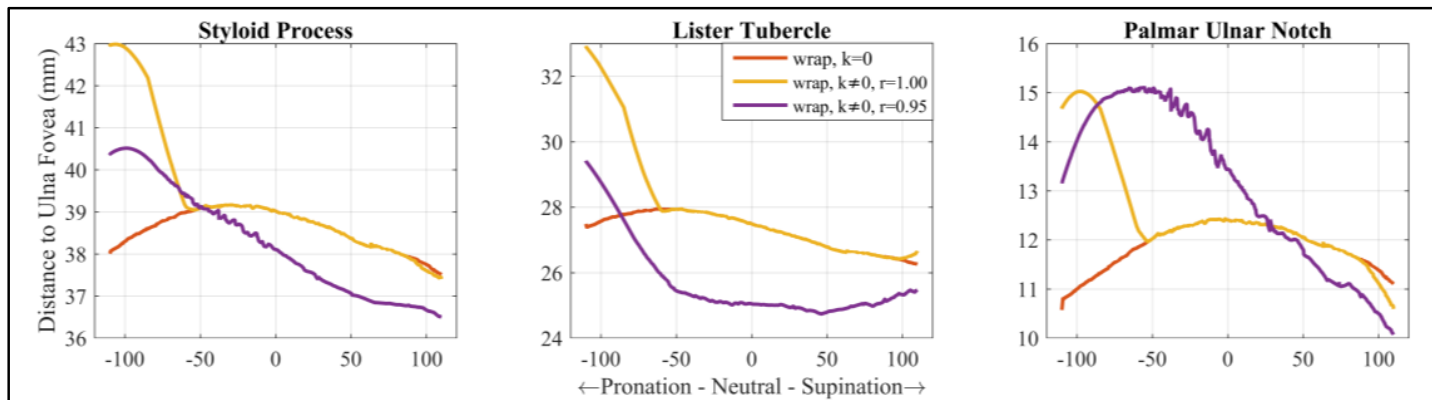

Figure 4: Position of three landmarks on the distal radius over the movement. 
Next, a second analysis has been performed. We force the radius to follow the ideal axis of rotation in a kinematics manner. Additionally, we record the forces acting on the radius. The forces are expressed in the radius frame as pictured in Figure 1. Scarring of the tissues in the IOM is a common observed phenomenon, however its pathogenesis and its effects remain unclear. We simulate it in our system by increasing the stiffness values by three: for the proximal part (POB, DOAC), middle part (CB), and distal part (DOB, AB) of the IOM, while keeping a rest length of $98 \%$ of the maximum length $(r=0.98)$. The results are shown in Figure 5.

We can notice that proximal scarring has a strong impact on the pronation by pushing to release the distal radioulnar joint $(\mathrm{Fx}>0)$ and pulling the radius toward the humerus $(\mathrm{Fz}<0)$. Elsewhere in the motion, for all cases, the IOM tends to help maintaining a prestressed contact with the cartilage $(\mathrm{Fx}<0)$. The central scarring of the central band greatly pulls the radius toward the wrist $(\mathrm{Fz}>0)$ and increases the contact with the cartilage $(\mathrm{Fx}<0)$. During supination, the ligaments resist to the desired motion $(\mathrm{Fy}>$ $0)$ or participate to generate the motion $(\mathrm{Fy}<0)$, and do the opposite in pronation, resisting $(\mathrm{Fy}<0)$ or participate $(\mathrm{Fy}>0)$.
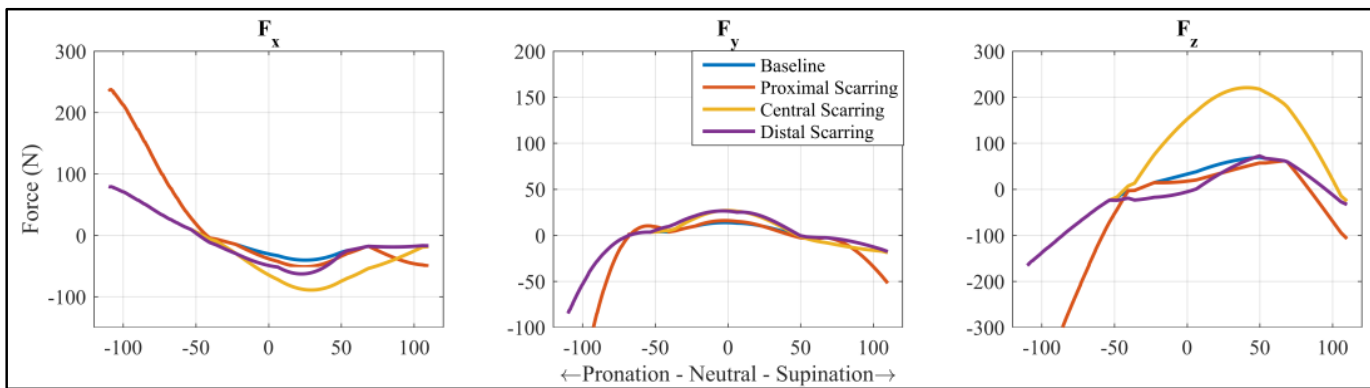

Figure 5: Effect of scarring on the forces generated by the ligaments on the Radius. Scarring is simulated by increasing threefold the stiffness of the ligaments: for the proximal (POB, DOAC), central (CB), and distal (DOB, AB) parts. Rest length is set at $98 \%$ of maximum length $(r=0.98)$.

\section{DISCUSSION}

The wrapping of the POB around the ulna is apparent as it is curving around the proximal radial tuberosity. However, our analysis shows that wrapping of the DOB around the ulna may arise at the limits of the pro-supination. The occurrence and influence of this phenomenon could be related to the anatomy, thus being patient dependent.

Previous studies (Pfaeffle 1996, Noda 2009) on the IOM anatomy or structural properties report mainly the stiffness, the thickness, and the width of the ligaments. 
However, they do not report the rest lengths. Our results indicate that they play a major role in the outcome and cannot be neglected for the physical simulation of the IOM.

The main benefit of our method consists reporting the effect of the kinetics of the ligaments, which can be analyzed to establish hypothesis concerning the causes of pathologies and the limitations of range of motion encountered by a patient that a kinematic method cannot, such as the scarring of tissues. In our simulation, we could see the resulting forces of the ligaments acting on the radius. A major result from it is that at around 60-degree supination, the system goes under a major regime shift. The force components induced by the ligaments maintaining the contact at the distal joint becomes small, while the ligaments become generator in supination and the radius is strongly pulled toward the wrist. The combination of these elements would indicate that a phenomenon such as subluxation is more likely to occur in that range. Further experiments will be required to evaluate thoroughly this hypothesis.

We modelled the radioulnar joint including the IOM and simulated the kinetic system such that the ligaments influence the path of the radius. Previous models (Kasten 2002, Yasutomi 2002) generally omit this physical IOM behavior, solving it purely geometrically without wrapping or material constraints, which potentially limits the range of pathologies that can be simulated. For example, the pathological cases where the IOM might be the reason for limited range of motion or instability could only be simulated and potentially diagnosed with our proposed system. Application to pathological cases and comparison to clinical observations is the subject of a future work.

\section{ACKNOWLEDGMENT}

This research has been funded by a Highly Specialized Medicine grant (HSM2) of the Canton of Zurich, Switzerland.

\section{REFERENCES}

- Fürnstahl P, Schweizer A, Nagy L, Székely G, \& Harders M, A morphological approach to the simulation of forearm motion, Proceedings of the 31st Annual International Conference of the IEEE Engineering in Medicine and Biology Society: Engineering the Future of Biomedicine, EMBC 2009, 7168-7171, 2009.

- Fürnstahl P, Computer-assisted planning for orthopedic surgery, Konstanz: Hartung-Gorre Verlag, 2010. 
- Kasten P, Krefft M, Hesselbach J, \& Weinberg A M, Computer simulation of forearm rotation in angular deformities: A new therapeutic approach, Injury, 33(9), 807-813, 2002.

- Lloyd J E, Stavness I, \& Fels S, ArtiSynth: A Fast Interactive Biomechanical Modeling Toolkit Combining Multibody and Finite Element Simulation, Soft Tissue Biomechanical Modeling for Computer Assisted Surgery, 355-394, 2012.

- Moritomo H, Noda K, Goto, A, Murase T, Yoshikawa H, \& Sugamoto K, Interosseous Membrane of the Forearm: Length Change of Ligaments During Forearm Rotation. The Journal of Hand Surgery, 34(4), 685-691, 2009.

- Murase T, Oka K, Moritomo H, Goto A, Yoshikawa H, \& Sugamoto K, ThreeDimensional Corrective Osteotomy of Malunited Fractures of the Upper Extremity with Use of a Computer Simulation System, The Journal of Bone \& Joint Surgery, 90(11), 2375 LP-2389, 2008.

- Noda K, Goto A, Murase T, Sugamoto K, Yoshikawa H, \& Moritomo H, Interosseous Membrane of the Forearm: An Anatomical Study of Ligament Attachment Locations, Journal of Hand Surgery, 34(3), 415-422, 2009.

- Pfaeffle H J, Stabile K J, Li Z M, \& Tomaino M M, Reconstruction of the interosseous ligament restores normal forearm compressive load transfer in cadavers. Journal of Hand Surgery, 30(2), 319-325, 2005.

- Pfaeffle H J, Tomaino M M, Grewal R, Xu J, Boardman N D, Woo S L, \& Herndon J H, Tensile Properties of the Interosseous Membrane of the Human Forearm, 842-845, 1996.

- Tarr R R, Garfinkel A I, \& Sarmiento A, The effects of angular and rotational deformities of both bones of the forearm. An in vitro study. The Journal of Bone \& Joint Surgery, 66(1), 65 LP-70, 1984.

- Vlachopoulos L, Schweizer A, Graf M, Nagy L, \& Fürnstahl P, Threedimensional postoperative accuracy of extra-articular forearm osteotomies using CT-scan based patient-specific surgical guides. BMC Musculoskeletal Disorders, 16(1), 336, 2015.

- Yasutomi T, Nakatsuchi Y, Koike H, \& Uchiyama S, Mechanism of limitation of pronation/supination of the forearm in geometric models of deformities of the forearm bones. Clinical Biomechanics, 17(6), 456-463, 2002.

\section{DISCLOSURES}

The authors have no financial conflicts of interest to disclose. 